\title{
IIIness perception, religiosity and mental health of diabetic patients in Ghana
}

\author{
Kingsley Nyarko, Nuworza Kugbey, Samuel Atindanbila \\ Psychology Department, University of Ghana, Legon, Melbourne
}

\section{Email address:}

kingpong73@yahoo.com(K. Nyarko),nkugbey@gmail.com(N. Kugbey), atindanbila@gmail.com(S. Atindanbila)

\section{To cite this article:}

Kingsley Nyarko, Nuworza Kugbey, Samuel Atindanbila. Illness Perception, Religiosity and Mental Health of Diabetic Patients in Ghana. American Journal of Applied Psychology. Vol. 3, No. 1, 2014, pp. 12-20. doi: 10.11648/j.ajap.20140301.13

\begin{abstract}
This study examines the influence of diabetic patients' perception of their illness and their levels of religiosity on their mental health problems. A sample of 194 diabetic patients was drawn from two major hospitals (Korle-Bu Teaching and Tema General Hospitals) in the Greater Accra Region of Ghana. The cross-sectional survey method was used as the study design. Results from Pearson correlation show that the diabetic patients' level of religiosity did not significantly correlate with their mental health problems. However, illness perception correlates significantly and positively with their general mental health problem (GSI) and specific ones such as somatization, obsessive-compulsion, depression, anxiety and psychoticism. Multiple regression analyses show that level of general mental health problem (GSI) was significantly predicted by perception of illness Coherence followed by perceptions Symptoms and Concern. Similarly, perception of coherence was the most significant predictor of both depression and anxiety among diabetic patients. The implications of the findings are discussed.
\end{abstract}

Keywords: Illness Perception, Religiosity, Mental Health, Diabetes, Ghana

\section{Introduction}

Diabetes can be defined as a situation where an individual's body is incapable of producing the hormone insulin in levels required by the body cells to take up optimal glucose (Kumar \& Clark, 2005). This chronic illness is characterized by gross weight loss, frequent urination, excessive thirst, and slow healing of wounds. As a result, if the condition is not well managed, it can lead to severe complications such as loss of blood circulation to heart and limb (Darkwa, 2011). These complications further pose serious mental health challenges to the patients as they have to adjust to the experiences of living with diabetes.

There are several diabetes related complications including both physical and mental health problems. For instance, diabetes has been found to be associated with reduced life-expectancy (Hall, Thomsen, Henriksen \& Lohse, 2011; Kengne, Amoah \& Mbanya, 2005). Stroke, hypertension, amputation, nephropathy, neuropathy, retinopathy, impotence and skin lesion (Langat, 2011), musculoskeletal problems (Kim, Edelman \& Kim, 2001) and coronary heart diseases (Barreto, Passos, Almeida \& Assis, 2007) are all some of the medical complications.
On the other hand, several mental health problems are found to be common among diabetic patients. Depression and anxiety (Lin \& von Korff, 2008; Coker, Ohaeri, Lawal \& Orija, 2000; Tuncay, Musabak, Gok \& Kutlu, 2008; Roupa et al., 2009), poor psychological wellbeing (Peyrot et al., 2004), affective disorders (Hermanns, Kulzer, Krichbaum, Kubiak \& Haak, 2004), sexual dysfunction (Coker, Ohaeri, Lawal \& Orija, 2000), irritability, anxiety, depression, suicidal ideas and cognitive deficits (Blanz, Rensch - Reimann, Fritz-Sigmund \& Schmidt, 1993) and eating disorders are some of the common mental health problems reported in diabetic population relative to the mental health of the general population. The issue however, is that when there are mental health comorbidities among diabetic patients, they affect other aspects of the management regimen such as adherence, self-care and severe physical symptoms (Hamdan, Tamim, Mahmood \& Young, 2010).

In sub-Saharan Africa, chronic illnesses are on the increase, however, growth rates of diabetes mellitus (DM) and hypertension are among the highest chronic diseases worldwide (Danquah et al., 2012). In Ghana, it is estimated that 4million people are living with diabetes and this number is expected to rise in the near future (National 
Diabetes Association of Ghana, 2012). Thus, several people are living with diabetes and its attendant complications. It is therefore believed that by 2025 , more than $75 \%$ of the world population with diabetes will reside in developing countries and the countries with the largest populations of adults with diabetes will include India, China and the United States (King, Aubert, \& Herman, 1998). Similarly, a recent study estimated diabetes to increase significantly by 2030 (Shaw, Sicree \& Zimmet, 2010).

In this study, the Self-Regulatory Model (Leventhal, Meyer \& Nerenz, 1980) and the Religious Coping theory (Pargament, 1997) were utilized to put the study in perspective. The Self- Regulatory Model (Leventhal, Meyer \& Nerenz, 1980; Leventhal et al., 1984; Leventhal et al., 1997; Leventhal et al. 2001) relates to perceptions that patients hold about their illnesses and as such the model posits that individuals actively generate cognitive and emotional representations of health threats and that these representations guide and regulate behaviour (Leventhal et al., 1997). Thus, the representation of illnesses in terms of the consequences, duration, symptoms, control, coherence and causes guide behaviour and influence health outcomes. The religious coping theory (Pargament, 1997) on the other hand, explains how individuals fall on their religious resources in dealing with threatening situation. The theory therefore, posits that, when people are faced with problems, they try to cope with their religious resources inherent in them which are likely to influence their health outcomes depending on the nature of their religiosity.

The experience of diabetes presents challenges to the patients and as such several psychosocial factors have been noted to influence the health outcomes of the patients. Some of these factors include personal religiosity, illness perception, coping strategies, social support as well as some demographic characteristics. This was emphasized by Leventhal, Weinman, Leventhal and Phillips (2008) that outcomes of medical management in patients with chronic illness are determined not only by objective factors, but also by behavioural and social factors.

People vary in how they perceive their health status and that these perceptions often are independent of the actual physical conditions that are being suffered (Taylor, Kemeny, Reed, Bower \& Gruenewald, 2000). For instance, people vary about how they perceive their possibilities to influence or control their health (Wallston, 2004), whether their condition is acute or chronic (Lau \& Hartman, 1983) or whether or not their specific situation is hopeful (Scheier \& Carver, 1985). Such perceptions may in turn determine individuals' behaviour as well as their response to managing health threats related to a disease or a symptom (Alsén, 2009).

Illness perception has been shown to have a significant influence on several health outcomes across illnesses and this is due to the fact that the interpretations given to the illness by patients affect how they live with their conditions. For example, illness perception has been shown to significantly influence adherence to insulin, cholesterol and antihypertensive medications, exercise, and diet (Broadbent, Donkin \& Stroh, 2011), body mass index, fasting blood glucose, total cholesterol and blood pressure (Petricek et al., 2009), illness distress (Paddison, Alpass \& Stephens, 2010) among diabetic patients. Similarly, illness perception significantly influenced health outcomes (psychological distress, depression, anxiety, quality of life, and adjustment) in other chronic illnesses such as oesophageal cancer (Dempster et al., 2011), interstitial cystitis (Heyhoe \& Lawton, 2009), Parkinson disease (Evans \& Norman, 2009), Breast cancer (Jørgensen, Frederiksen, Boesen, Elsass \& Johansen, 2009), allergy sufferers (Knibb \& Horton, 2008).

Furthermore, the individual's personal religiosity and organized religion are seen as protective factors for a person against the development of mental health problems. Several study outcomes have demonstrated consistent pattern of religiosity serving as a protective factor against development of mental health and physical health problems (e.g. Park et al., 2012; Abdoli et al., 2011; How, Ming \& Chin, 2011; Kilbourne, Cummings \& Levine, 2009; King, Mainous \& Pearson, 2002; Baetz et al., 2004; Abdoli, Ashktorab, Ahmadi, Parvizy \& Dunning, 2011). In a study among Canadian population by Baetz, Bowen, Jones and Koru-Sengul (2006), it was found that higher worship frequency is associated with lower odds of psychiatric disorders. In the same vein, Kilbourne, Cummings and Levine (2009) examined the influence of religiosity on depression among low-income people with diabetes in a mid-sized southern city in the US and reported that religious dimensions such as prayer, religious reading, religious attendance, and religious belief proved protective against depressive symptoms.

However, some studies did not find any significant influence of religiosity on mental health outcomes (e.g. Edmondson et al., 2005; Morse et al., 2000; Miller, McConnell \& Klinger, 2007). Nonetheless, some studies have also demonstrated the negative impact of religiosity/spirituality on health outcomes (e.g. Baetz et al., 2006; Park, Hong, Park \& Cho, 2012). These contradictory findings across studies create an opportunity for studies to be conducted to examine whether personal religious faith protects diabetic patients against mental health problems or otherwise. With regards to diabetic studies in Ghana, the few available ones are mainly prevalence studies that are concerned with estimation of number of people suffering from diabetes and the medical complications (e.g. Darkwa, 2011; Adubofour, Ofei, Mensah-Adubofour \& Owusu, 1993), illness experience and action (e.g. De-Graft Aikins, 2003), with no such studies addressing the mental health problems that are likely to be experienced by diabetic patients.

In the same vein, majority of the studies seem to have focused on how illness perception influences psychological distress, but not specific mental health problems and therefore, makes it difficult in determining the impact of the illness perceptions on specific psychological disorders. Similarly, most of the studies (e.g. McCabe, Barnason \& 
Houfek, 2011; Dempster et al., 2011; Heyhoe \& Lawton, 2009; Evans \& Norman, 2009) reviewed on illness perception focused on other conditions with not much attention given to diabetes mellitus even though the illness is estimated to rise in number in Ghana. Though Ghanaians are very religious (Gyekye, 1996), not much has been done to examine the influence of religiosity on specific mental health problems.

In line with the above reviewed studies, the study sought 1) to investigate whether the perception of diabetes by the patients significantly influence their mental health problems, 2) examine the relationship between diabetic patients' level of religiosity and their mental health problems, and 3) examine which of the illness perception components predict the levels of depression and anxiety among diabetic patients significantly.

Flowing from the above, it was thus hypothesized that 1) there will be a significant positive relationship between illness perception of diabetic patients and their mental health problems, 2) there will be a significant negative relationship between diabetic patients' religiosity and mental health problems, 3) the diabetic patients' perception of illness coherence is likely to account for more variance in mental health problem (GSI) than the other illness perception components, 4) the diabetic patients' perception of illness coherence is likely to account for more variance in their level of depression than the other illness perception components, and 5) the diabetic patients' perception of illness coherence is likely to account for more variance in their level of anxiety than the other illness perception components.

\section{Methodology}

\subsection{Population and Sample}

All diabetic patients receiving out-patients care at Korle$\mathrm{Bu}$ Teaching and Tema General Hospitals. These hospitals were chosen because they serve the referral points for varied clients within the Accra Metropolitan-and Tema Metropolitan Assemblies. The convenient sampling technique was used to select one hundred and ninety four (194) participants representing a 97\% response rate. Out of the sample size of 194,46 of them representing $24 \%$ are male patients and 148 of them representing $76 \%$ are female patients. For the age categories of the patients, 6 of them representing 3\% are between 20-29 years, 10 of them representing $5 \%$ are between $30-39$ years, 29 of them representing $15 \%$ are between $40-49$ years, 60 of them representing $31 \%$ are between $50-59$ years and 89 of them representing $46 \%$ are above the age of 60 years. In terms of respondents' marital status, 20 of them representing $10 \%$ are single, 117 representing $60 \%$ are married, 17 representing $9 \%$ are separated/ divorced and 40 representing $21 \%$ are widows

For the level of education of the participants, 22 representing $11 \%$ have no formal education, 98 representing
$51 \%$ have primary education, 52 representing $27 \%$ have secondary education and 22 representing $11 \%$ have tertiary education respectively. For their religious affiliations, 176 representing $91 \%$ are Christians, 16 representing $8 \%$ are Muslims and 2 representing 1\% belong to other religions. There were 50 Type- 1 diabetic patients representing $26 \%$ and 144 Type-2 diabetic patients representing 74\%. For the illness duration, a mean score of 8.69 years with a standard deviation of 7.30 years were obtained.

\subsection{Measures}

A set of questionnaires and inventories was employed as the main measure for the study. The first section was made up of respondents' demographic characteristics followed by questionnaires and inventories.

\subsubsection{Brief Illness Perception Questionnaire (Broadbent, Petrie, Main, \& Weinman, 2006)}

This is a nine-item instrument which measures patients' cognitive and emotional representations of their illness including their perceptions of illness consequences, duration, personal control, treatment control, symptoms, coherence, concern, emotional response, and causes. Examples of items include, "how much control do you feel you have over your illness?" (Personal control), "how long do you think your illness will continue?" (Timeline) and, "how much does your illness affect your life?" (Consequence). The causal item was open-ended to allow respondents to indicate what they thought caused their illness. A Cronbach alpha of 0.70 was reported for the scale. This scale also demonstrated good concurrent validity with relevant measures, predictive validity and discriminate validity (Broadbent, Petrie, Main, \& Weinman, 2006). An overall illness perception score is computed to determine whether the illness is viewed as benign or threatening, the authors suggested that items 3, 4, and 7 should be reverse scored and added to items 1, 2, 5, 6, and 8. A higher score reflects a more threatening view of the illness.

\subsubsection{Santa Clara Strength of Religious Faith Questionnaire (Plante \& Boccaccini, 1997)}

This is a ten-item instrument which measures an individual's level of religious faith. Some examples of items on the scale are "I pray daily," "I look to my faith as a source of inspiration," and "I look to my faith as providing meaning and purpose in my life." This scale has a likert response format of 4-points including; "1 = strongly disagree," "2 = disagree," "3 = agree," "4 = strongly agree." Studies that have investigated the internal consistency of the scale have found corrections ranging from 0.94 to 0.97 using Cronbach Alpha's and split-half reliability scores ranging from 0.90 to 0.96 (Plante \& Boccaccini, 1997). The total religiosity score is obtained for each respondent by adding the responses on all the ten items with a maximum score of 40 and a minimum score of 10 with higher scores representing a higher level of religiosity and vice versa. 


\subsubsection{Brief Symptom Inventory (Derogatis, 1993)}

The Brief Symptom Inventory is a 53-item self-report symptom inventory designed to reflect the psychological symptom patterns of psychiatric and medical patients and non-patients. This inventory reports profiles of nine primary symptom dimensions and three global indices of distress (Derogatis, 1993). The symptom dimensions included somatization, obsessive-compulsive, interpersonal sensitivity, depression, anxiety, hostility, phobic anxiety, paranoid ideation and psychoticism. The responses are on a 5-point scale, ranging from $0=$ "not at all" to $4=$ "extremely". The BSI also has high internal consistency (Cronbach's alpha: 0.71-0.85), test retest reliability, and convergent, discriminant, and construct validity.

\subsection{Procedure}

Ethical clearance was obtained from the Institutional Review Board of the Noguchi Memorial Institute for Medical Research, University of Ghana, Legon. Approval was obtained from the Greater Accra Regional Health Directorate for permission to use Tema General Hospital. For the Korle-Bu Teaching Hospital, a completed Diabetes Research Form and Ethical clearance certificate were sent to the Head of the Diabetic Centre at Korle-Bu for approval and permission.

Two research assistants were recruited for the data collection and were given training on administration of the questionnaires. The out- patients departments of the hospitals were used for the data collection. On the days of the data collection, the researchers were introduced to the patients waiting to see their doctors after which the researchers and the two assistants engaged the patients individually. The patients were given the consent form to write their names and sign or make a mark indicating their voluntary participation. The respondents who could read and write were administered the questionnaires in a penand paper form. Those who could not were interviewed by following the questions on the questionnaires. During the data collection, those who could not finish were given the chance to go and their questionnaires were not included in the analysis. The data collection lasted for five weeks.

\subsection{Data Analysis}

The main hypotheses for the study were analyzed by using inferential statistics. Hypotheses 1 and 2 were analyzed using the Pearson correlation because the researchers sought to find out the relationship between the variables. Specifically, the researchers sought to find out the relationship between religiosity and mental health of the patients, as well as the relationship between illness perception and mental health problem. These hypotheses are correlational, and thus a relational statistical intervention is the most appropriate.

The hypotheses $3,4, \& 5$ were tested with multiple regression analysis because illness perception components (Consequences, Duration, Personal Control, Treatment Control, Symptoms, Concern, Coherence and Emotional Response) were regressed on mental health problem (GSI), depression and anxiety respectively.

\section{Results}

\subsection{Hypotheses Testing}

To test hypotheses 1 and 2 which sought to establish relationships between illness perception and mental health problems on one hand, and religiosity and mental health problems on the other hand, the Pearson correlation was done and presented in the correlation matrix table 1 below.

Table 1. Correlation Matrix of Illness Perception, Religiosity and Mental Health Problems of Diabetics Patients

\begin{tabular}{|c|c|c|c|c|c|c|c|c|c|c|c|}
\hline VAR & 1 & 2 & 3 & 4 & 5 & 6 & 7 & 8 & 9 & 10 & 11 \\
\hline \multicolumn{12}{|l|}{ REL } \\
\hline PERC & $-.13^{*}$ & & & & & & & & & & \\
\hline SOM & .06 & $.20 * *$ & & & & & & & & & \\
\hline OC & .07 & $.13 *$ & $.58 * *$ & & & & & & & & \\
\hline SEN & .08 & .08 & $.51^{* *}$ & $.47 * *$ & & & & & & & \\
\hline DEP & .05 & $.15^{*}$ & $.52 * *$ & $.54 * *$ & $.60 * *$ & & & & & & \\
\hline ANX & .10 & $.19 * *$ & $.66^{* *}$ & $.59 * *$ & $.54 * *$ & $.54 * *$ & & & & & \\
\hline HOS & .03 & .10 & $.50 * *$ & $.51^{* *}$ & $.54 * *$ & $.57 * *$ & $.45^{* *}$ & & & & \\
\hline PHO & .05 & .11 & $.43 * *$ & $.56^{* *}$ & $.46^{* *}$ & $.52 * *$ & $.61^{* *}$ & $.35^{* *}$ & & & \\
\hline PAR & .07 & -.06 & $.29 * *$ & $.26^{* *}$ & $.47 * *$ & $.31 * *$ & $.24 * *$ & $.35 * *$ & $.25 * *$ & & \\
\hline PSY & .08 & $.12 *$ & $.37 * *$ & $.43 * *$ & $.57 * *$ & $.60 * *$ & $.41^{* *}$ & $.45^{* *}$ & $.42 * *$ & $.50^{* *}$ & \\
\hline GSI & .09 & $.22 * *$ & $.74 * *$ & $.74 * *$ & $.73^{* *}$ & $.75 * *$ & $.77 * *$ & $.67^{* *}$ & $.66^{* *}$ & $.50^{* *}$ & $.67^{* *}$ \\
\hline
\end{tabular}

$* *=$ Significant at .01 alpha level, *= significant at .05 alpha level. REL=Religiosity, PERC=Illness Perception, SOM=Somatization, OC=ObsessiveCompulsion, SEN=Interpersonal Sensitivity, DEP= Depression, ANX= Anxiety, HOS=Hostility, PHO=Phobic-anxiety, PAR= Paranoid Ideation, PSY= Psychoticism and GSI= Global Severity Index. 
From the correlation matrix 1 above, a significant positive relationship was obtained between illness perception and Global Severity Index (general mental health problem), $r(192)=.22, \rho<.01$. Further analyses showed that illness perception correlated significantly with five (somatization, obsessive-compulsion, depression, anxiety and psychoticism) of the mental health problems, that is, somatization, $r(192)=.20, \rho<.01$, obsessivecompulsion, $r(192)=.13, \rho<.05$, interpersonal sensitivity, $r(192)=.08, \rho>.05$, depression, $r(192)=.15, \rho<.05$, anxiety, $r(192)=19, \rho<.01)$, hostility, $r(192)=.10, \rho>.05$, phobic anxiety, $r(192)=.11, \rho>.05$, Paranoid Ideation, $r(192)=-.01, \rho>.05)$, and Psychoticism, $r(192)=.12, \rho$ $<.05$. Therefore, the first hypothesis that there will be a significant positive relationship between Illness Perception of diabetic patients and their mental health problems is supported.

However, it was observed from the correlation matrix that religiosity does not significantly correlate with the Global Severity Index (general mental health problem), $\mathrm{r}(192)=.09, \rho>.05$. Further analysis showed no significant relationships between religiosity and specific mental health problems, that is, somatization; $r(192)=.06$, $\rho>.05$, obsessive-compulsion; $\mathrm{r}(192)=.07, \rho>.05$, interpersonal sensitivity; $\mathrm{r}(192)=.08, \rho>.05$, depression; $\mathrm{r}(192)=.05, \rho>.05$, anxiety; $\mathrm{r}(192)=.10, \rho>.05$, hostility; $r(192)=.03, \rho>.05$, phobic anxiety; $r(192)=.05$, $\rho>.05$, paranoid ideation; $r(192)=.07, \rho>.05$, and psychoticism $\mathrm{r}(192)=.08, \rho>$.05.Therefore, the hypothesis two that there will be a significant negative relationship between diabetic patients' religiosity and mental health problems is not supported.

Hypothesis Three: The perception of illness coherence will significantly predict the mental health problem (GSI) of diabetic patients than the other illness perception components.

Multiple regression analyses using the ENTER method was used to find out how the various components of the illness perception predict the mental health problems (Global Severity Index) among diabetic patients. A significant model emerged at the .001 alpha level, $\left[R^{2}=.33\right.$, $F(8,185)=11.16, \rho<.001]$. That is the entire model explained about $33 \%$ of variance in the level of general mental health problem (GSI) among diabetic patients. The contributions of each of the illness perception components in explaining the variance in general mental health problem are summarised in the table 2 below.

Table 2. Multiple Regressions of the contributions of the Components to Global Severity Index

\begin{tabular}{llllll}
\hline Predictors & $\mathbf{B}$ & SEB & $\boldsymbol{\beta}$ & $\mathbf{t}$ & $\boldsymbol{\rho}$ \\
\hline Consequences & .01 & .01 & .06 & .81 & .417 \\
Duration & .02 & .01 & .10 & 1.50 & .135 \\
Personal Control & .00 & .02 & .00 & .01 & .990 \\
Treatment Control & .00 & .02 & .01 & .08 & .933 \\
Symptoms & .06 & .02 & .27 & 3.89 & .000
\end{tabular}

\begin{tabular}{llllll}
\hline Predictors & B & SEB & $\boldsymbol{\beta}$ & t & p \\
\hline Concern & .03 & .01 & .17 & 2.46 & .015 \\
Coherence & -.05 & .01 & -.33 & -4.92 & .000 \\
Emotional Response & .01 & .01 & .07 & .97 & .336 \\
\hline
\end{tabular}

Predictors: Consequences, Duration, Personal Control, Treatment Control, Symptoms, Concern, Coherence and Emotional Response.

An examination of Table 2 above showed that the most significant illness perception component predictor of Global Severity Index is the perception of coherence which contributed negatively to $33 \%$ of variance in psychological distress at the .01 alpha level, $[\beta=-.33, t=-4.92, \rho<.01]$. The second most significant predictor of psychological distress (GSI) was the perception of Symptoms of the illness which contributed positively to $27 \%$ of variance in psychological distress at the .01 alpha level, $[\beta=.27, t=$ $3.89, \rho<.01]$. The third most significant predictor of general psychological distress (GSI) was perception of Concern about the illness which contributed positively to $17 \%$ of variance in psychological distress at the .05 alpha level, $[\beta=.16, \mathrm{t}=2.46, \rho<.05]$. The other illness perception components did not predict the level of psychological distress (GSI) significantly as shown in the multiple regressions table 2 above. Thus, the third hypothesis that perception of illness coherence will significantly predict the mental health problem (GSI) of diabetic patients than the other illness perception components is supported.

Hypothesis Four: The perception of illness coherence will significantly predict the level of depression among diabetic patients than the other illness perception components.

Multiple regression was done and a significant model emerged at the .001 alpha level, $\left[R^{2}=.27, F(8,185)=8.55\right.$, $\rho<.001]$. That is the entire model explained about $27 \%$ of variance in the level of depression among diabetic patients. The contributions of each of the illness perception components in explaining the variance in the level of depression are summarized in the table 3 below.

Table 3. Multiple Regression of the contributions of illness perception components to depression

\begin{tabular}{lccccc}
\hline Predictors & $\mathbf{B}$ & SEB & $\boldsymbol{\beta}$ & $\mathbf{t}$ & $\boldsymbol{\rho}$ \\
\hline Consequences & .01 & .01 & .07 & .88 & .379 \\
Duration & .02 & .01 & .14 & 2.00 & .047 \\
Personal Control & .01 & .02 & .03 & .35 & .728 \\
Treatment Control & .00 & .02 & .00 & .03 & .973 \\
Symptoms & .04 & .02 & .16 & 2.16 & .032 \\
Concern & .01 & .01 & .06 & .83 & .407 \\
Coherence & -.05 & .01 & -.30 & -4.28 & .000 \\
Emotional Response & .04 & .01 & .21 & 2.71 & .007 \\
\hline
\end{tabular}

Predictors: Consequences, Duration, Personal Control, Treatment Control, Symptoms, Concern, Coherence and Emotional Response. 
From the Table 3 above, it was observed that the most significant illness perception component in predicting the level of depression among diabetic patients is the perception of illness coherence, $[\beta=-.30, t=-4.28, \rho$ $<.001]$. This was followed by the perception of emotional response, $[\beta=.21, \mathrm{t}=2.71, \rho<.01]$, perception of symptoms of the illness $[\beta=.16, t=2.16, \rho=.05]$ and perception of duration $[\beta=.14, t=2.00, \rho<.05]$ respectively. The perceptions of consequences, personal control, treatment control and concern did not however, account for any significant variance in the level of depression among diabetic patients. Therefore, the hypothesis that the perception of illness coherence will significantly predict the level of depression among diabetic patients than the other illness perception components is supported.

Hypothesis Five: The perception of illness coherence will significantly predict the level of anxiety among diabetic patients than the other illness perception components.

The multiple regression was done and a significant model emerged at the .001 alpha level, $\left[R^{2}=.25, F(8,185)=\right.$ $7.50, \rho<.001]$. That is, the entire model explained about $25 \%$ of variance in the level of anxiety among diabetic patients. The contributions of each of the illness perception components in explaining the variance in the level of anxiety are summarized in the table 4 below:

Table 4. Multiple Regression of the contributions of illness perception components to Anxiety

\begin{tabular}{lccccc}
\hline Predictors & $\mathbf{B}$ & $\mathbf{S E B}$ & $\boldsymbol{\beta}$ & $\mathbf{t}$ & $\boldsymbol{\rho}$ \\
\hline Consequences & .01 & .02 & .03 & .34 & .738 \\
Duration & .02 & .02 & .11 & 1.53 & .129 \\
Personal Control & -.02 & .03 & -.05 & -.59 & .554 \\
Treatment Control & -.01 & .03 & -.03 & -.35 & .729 \\
Symptoms & .07 & .02 & .24 & 3.22 & .002 \\
Concern & .02 & .02 & .11 & 1.53 & .128 \\
Coherence & -.07 & .02 & -.29 & -4.16 & .000 \\
Emotional Response & .01 & .02 & .04 & .53 & .597 \\
\hline
\end{tabular}

Predictors: Consequences, Duration, Personal Control, Treatment Control, Symptoms, Concern, Coherence and Emotional Response.

An examination of Table 4 above shows that the most significant illness perception of anxiety among diabetic patients is the perception of illness coherence which explained $29 \%$ of variance in anxiety, $[\beta=-.29, t=-4.16, \rho$ $<.001]$. This was followed by the perception of symptoms which explained $24 \%$ of variance in Anxiety, $[\beta=.24, t=$ $3.22, \rho<.01]$. The perceptions of consequences, duration, personal control, treatment control, concern and emotional response did not significantly predict the level of anxiety among diabetic patients. Therefore, the hypothesis that the perception of illness coherence will significantly predict the level of anxiety among diabetic patients than the other illness perception components is supported.

\section{Discussion and Recommendations}

To examine whether illness perception of diabetic patients significantly relates with their levels of mental health problems, it was observed that significant positive relationships exist between illness perception and general mental health problem (GSI). Illness perception was also found to be significantly related to some specific mental health problems such as levels of somatization, obsessivecompulsion, depression and anxiety. This is because when the diabetes is perceived as threatening, it is likely to induce certain emotional feelings that are usually negative leading to the individual becoming depressed, anxious and psychologically distressed. That is the cognitive appraisal of the illness generates emotional, physiological and behavioral reactions that negatively influence the mental and physical health of the individual living with diabetes mellitus.

The finding of a significant influence of illness perception on the levels of somatization, depression, anxiety, and psychological distress among diabetic patients is consistent with previous works on illness perception and health outcomes. For instance, illness perceptions accounted for most of the variance in health outcomes compared to demographic variables, clinical history and extent of disease (Fortune, Richards, Griffiths \& Main, 2002). Similarly, illness perception significantly predicted distress among cancer sufferers (Rees, Fry, Cull \& Sutton, 2004), distress among allergy sufferers (Knibb \& Horton, 2008), anxiety and depression among Parkinson's disease patients (Evans \& Norman, 2009), psychological distress among patients with interstitial cystitis(IC) (Heyhoe \& Lawton, 2009). In the same vein, Paddison, Alpass and Stephens (2010) found illness perception to have significantly predicted diabetes-related distress.

The second hypothesis that stated that there will be a significant negative relationship between diabetic patients' religiosity and mental health problems was not supported. It was found that the level of religiosity is not significantly related to the mental health problems of diabetic patients and therefore did not significantly predict any of the mental health problems experienced by the diabetic patients. The lack of a significant relationship between the levels of diabetic patients' religiosity and their mental health problems is likely due to the fact that almost all of the patients reported high levels of religiosity which cut across and do not differ significantly in their levels of religiosity. As a result, religiosity becomes a normal part of life of these patients which reflects the general religious nature of the Ghanaian as religion is seen as an integral part of the majority of the Ghanaian populace (Gyekye, 1996). Another reason could be due to the fact that the various aspects of religiosity were not explored to determine which one significantly influences the levels of mental health problems of diabetic patients.

This finding is incongruent with earlier studies that showed significant influences of religiosity on several aspects of health including elevated C-reactive protein 
among diabetic patients (King, Mainous \& Pearson, 2002), depression (Baetz et al., 2004; Kilbourne, Cummings \& Levine, 2009; Park, et al., 2012), empowerment (Abdoli et al., 2011), glycaemic control among diabetic patients (How, Ming \& Chin, 2011). In all these previous studies, the level of religiosity was found to have significant influence on these outcomes among different samples in different countries by serving as a protective factor against health problems.

Furthermore, the level of depression experienced by diabetic patients was significantly predicted by perceptions of illness coherence followed by emotional response, symptoms and duration respectively. That is when there is a better understanding of the illness, the level of depression decreases as perception of illness coherence accounted for a significant decrease in the level of depression. This is because when the individual understands the illness, it is easier to cope in terms of treatment and the management of the condition. This finding supports previous findings that reduced illness coherence predisposes the individual to experience depression (Leventhal, Leventhal \& Cameron, 2001; Hermele et al., 2007).

Similarly, the emotional response to the illness that is, being sad or angry results in higher levels of depression among diabetic patients. The perception of many severe symptoms was also associated with higher levels of depressions as the experience of more symptoms by the patients results in emotional reactions such as sadness which predispose the individual to depression. In the same vein, perceived duration was found to significantly predict the level of depression which means that as the individual perceives diabetes to last forever, the level of depression also increases. However, a perception of a shorter duration of diabetes is associated with lower levels of depression among diabetic patients. This implies that thoughts about how long the illness will last play a significant role in the emotional reactions to diabetes by diabetic patients. Similar findings were documented by Heyhoe and Lawton (2009) and Evans and Norman (2009) that emotional representation predicted the level of depression.

More so, the level of anxiety experienced by the diabetic patients was significantly predicted by perception of illness coherence followed by perception of symptoms. That is when the individual perceives that he or she understands the illness, the anxiety associated with the illness decreases. Thus, when the individual has an insight into the illness and how to manage it very well, the fear associated with diabetes is likely to be reduced. In terms of the symptoms, perception of many severe symptoms results in anxiety as many symptoms signify diabetes-related complications which could even result in amputation. Thus, when the diabetes patients perceive more symptoms they are predisposed to the experience of anxiety which can interfere with their treatment regimen.

This finding concurs with the work of Petriček et al. (2009) that the understanding of diabetes was associated with high blood pressure which can induce the feeling of anxiety. Similar findings were reported by Dempster et al. (2011), McCabe, Barnason and Houfek (2011) and Paddison, Alpass and Stephens (2010). Diabetes, as an illness has several symptoms and complications which when not well understood could be major sources of worry and fear among diabetic patients thereby highlighting the significance of perception of illness coherence in predicting anxiety among diabetic patients.

The findings from this study have implications for diabetes management in Ghana at the individual, professional as well as institutional levels. For the individual diabetic patients, since religiosity was not found to be significantly related with their mental health problems unlike illness perception, conscious efforts should be made at following treatment regimen and positive reappraisal of their illness to reduce the distress associated with negative emotional and cognitive reactions. At the professional level, mental health professionals dealing with diabetic patients should take into cognizance the beliefs and perceptions of their patients about their illness and factor them into their management plans. The Ghana Health Service should take concrete steps to employ more mental health professionals such as clinical psychologists to form a holistic team in the management of diabetes. The shift from the focus on biomedical model of treatment to the bio-psychosocial model would be a beneficial one to all parties involved in dealing with chronic illnesses such as diabetes.

\section{Conclusion}

As the prevalence of diabetes has been shown to be high and is expected to increase in the near future in Ghana and elsewhere, the health system is faced with challenges regarding the management of the condition. Coupled with the fact that living with diabetes has several implications for the individual and the nation as a whole, the comorbid mental health problems have been shown to influence the management of the condition. It is therefore, imperative that the factors affecting these mental health problems are identified. As has been demonstrated across studies among different illnesses including cancer, asthma, allergy, diabetes, Myocardial Infarction and Parkinson disease among others, illness perception by patients was shown to be a significant predictor of overall mental health problems as well as the level of depression and anxiety experienced by diabetic patients. On the whole, the perception of illness coherence was found to be the most significant predictive component and as such informs healthcare professionals for the need for Psychoeducation on chronic illness to reduce the level of distress among patients.

\section{References}

[1] Abdoli, S., Ashktorab, T., Ahmadi, F., Parvizy, S., \& Dunning, T. (2011). Religion, faith and the empowerment process: Stories of Iranian people with diabetes. International Journal of Nursing Practice, 17, 289-298. 
[2] Adubofour, K. O. M., Ofei, F., Mensah-Adubofour, J., \& Owusu, S. K. (1993). In De-Graft Aikins, A. (Ed.). Living with Diabetes in Rural and Urban Ghana: A Critical Social Psychological Examination of Illness Action and Scope for Intervention. Journal of Health Psychology, 8(5) 557-572.

[3] Alsén, P. (2009). Illness Perception and Fatigue after Myocardial Infarction. An unpublished thesis, University of Gothenburg.

[4] Baetz, M., Bowen, R., Jones, G., \& Koru-Sengul, T. (2006). How spiritual values and worship attendance relate to psychiatric disorders in the Canadian population. Canadian Journal of Psychiatry, 51(10), 654-661.

[5] Baetz, M., Griffin, R., Bowen, R. C., Koenig, H. G., \& Marcoux, G. (2004). The association between spiritual/religious commitment and depressive symptoms in the Canadian population. Journal of Nervous and Mental Disease, 192(12), 818-822.

[6] Barreto, S. M., Passos, V. M. A., Almeida, S. K. F., \& Assis, T. D. (2007). The increase of Diabetes Mortality Burden among Brazilian Adults. American Journal of Public Health, 22(4), 239-245.

[7] Blanz, B., Rensch-Riemann, B., Fritz-Sigmund, D., et al. (1993). IDDM is a risk factor for adolescent psychiatric disorders. Diabetes Care, 16(12), 1579-1587.

[8] Broadbent, E., Petrie, K.J., Main, J., \& Weinman, J. (2006). The Brief Illness Perception Questionnaire (BIPQ). Journal of Psychosomatic Research, 60, 631-637.

[9] Coker, A. O., Ohaeri, J. U., Lawal, R. A., \& Orija, O. B. (2000). Specific Psychiatric Morbidity among Diabetics at a Nigerian General Hospital. East African Medical Journal, $77(1), 42-45$.

[10] Danquah, I., Bedu-Addo, G, Terpe, K., Micah, F., Amoako, Y. A., Awuku, Y. E., Dietz, E., van der Giet, M., Spranger, J., \& Mockenhaupt, F. P. (2012). Diabetes mellitus type 2 in urban Ghana: characteristics and associated factors. BMC Public Health, 12, 210-218.

[11] Darkwa, S. (2011). Prevalence of diabetes mellitus and resources available for its management in the Cape Coast Metropolis. Journal of Health and Environmental Sciences, 1(1), 1-7.

[12] De-Graft Aikins, A. (2003). Living with Diabetes in Rural and Urban Ghana: A Critical Social Psychological Examination of Illness Action and Scope for Intervention. Journal of Health Psychology, 8(5) 557-572.

[13] Dempster, M., McCorry, N. K., Brennan, E., Donnelly, M., Murray, L. J., \& Johnston, B. T. (2011). Psychological distress among family carers of oesophageal cancer survivors: the role of illness cognitions and coping. PsychoOncology, 20, 698-705.

[14] Derogatis, L.R. (1975). Brief Symptom Inventory. Baltimore: Clinical Psychometric Research.

[15] Edmondson, K. A., Lawler, K. A., Jobe, R. L., Younger, J. W., Piferi, R. L., \& Jones, W. H. (2005). Spirituality Predicts Health and Cardiovascular Responses to Stress in Young Adult Women. Journal of Religion and Health, 44(2), 161-171.

[16] Fortune, D. G., Richards, H. L., Griffiths, C. E. M., \& Main, C. J. (2002). Psychological stress, distress and disability in patients with psoriasis: Consensus and variation in the contribution of illness perceptions, coping and alexithymia. British Journal of Clinical Psychology, 41, 157-174.

[17] Hall, V., Thomsen, R. W., Henriksen, O., \& Lohse, N. (2011). Diabetes in Sub Saharan Africa 1999-2011: Epidemiology and public health implications. A systematic review. BMC Public Health, 11, 564-576.

[18] Hermele, S., Olivo, E. L., Namerow, P. \& Oz, M. C. (2007). Illness representations and psychological distress in patients undergoing coronary artery bypass graft surgery. Psychology Health Medicine, 12(5), 580-591.

[19] Heyhoe, J., \& Lawton, R. (2009). Distress in patients with interstitial cystitis: Do illness representations have a role to play? Psychology, Health \& Medicine, 14(6), 726-739.

[20] Jørgensen, I. L., Frederiksen, K., Boesen,E., Elsass, P., \& Johansen, C. (2009). An exploratory study of associations between illness perceptions and adjustment and changes after psychosocial rehabilitation in survivors of breast cancer. Acta Oncologica, 48, 1119-1127.

[21] Kengne, A. P., Amoah, A. G., \& Mbanya, J. C. (2005). Cardiovascular complications of diabetes mellitus in subSaharan Africa. Circulation, 112, 3592-3601.

[22] Kilbourne, B., Cummings, S. M., \& Levine, R. S. (2009). The Influence of Religiosity on Depression among LowIncome People with Diabetes. Health \& Social Work, 34(2), 137-147.

[23] King, H., Aubert, R., \& Herman, W. (1998). Global burden of diabetes, 1995-2025. Prevalence, numerical estimates and projections. Diabetes Care, 21, 1414-1431.

[24] King, D.E., Mainous, III, A.G., \& Pearson, W.S. (2002). Creactive protein, diabetes, and attendance at religious services. Diabetes Care, 25(7), 1172-1176.

[25] Knibb, R. C., \& Horton, S. L. (2008). Can illness perceptions and coping predict psychological distress amongst allergy sufferers? British Journal of Health Psychology, 13, 103-119.

[26] Kumar, P. \& Clark, M. (2005). Clinical Medicine (6th ed.) London: New York, Edinburgh Publishers.

[27] Langat, M. K. (2011). Prevention of Diabetes Mellitus Complications among Adults. An unpublished Bachelor's Thesis, Turku University of Applied Sciences.

[28] Lau, R. R. \& Hartman, K. A. (1983). Common sense representations of common illnesses. Health Psychology, $2,167-85$.

[29] Leventhal, H., Meyer, D., \& Nerenz, D. R. (1980). The common sense representation of illness danger. In Rachman, S. (Ed). Contributions to Medical Psychology. 2: 17-30. New York. Pergamon Press.

[30] Leventhal, H., Benyami, Y., Brownlee, S. Diefenbach., Leventhal, E.A, Patrick-Miller, L. and Robitaille, C. (1997). In Petrie, K. and Weinman, J. (Eds) Perceptions of health and illness. London. Harwood Press.

[31] Leventhal, H., Weinman, J., Leventhal, E. A., \& Phillips, L. A. (2008). Health psychology: The search for pathways between behaviour and health. Annual Review of Psychology, 59, 1-29. 
[32] Lin, E. H. B., \& von Korff, M. (2008). Mental disorders among persons with diabetes; Results from the World Mental Health Surveys, Journal of Psychosomatic Research $65,571-580$.

[33] McCabe, P. J., Barnason, S. A., \& Houfek, J. (2011). Illness Beliefs in Patients with Recurrent Symptomatic Atrial Fibrillation. PACE, 34, 810-820.

[34] Morse, E. V., Morse, P. M., Klebba, K. E., Stock, M. R., Forehand, R., \& Panayotova, E. (2000). The use of Religion among HIV-Infected African American Women. Journal of Religion and Health, 39(3), 261-276.

[35] Naemiratch, B. \& Manderson, L. (2007). Lay Explanations of Type 2 Diabetes in Bangkok, Thailand. Anthropology \& Medicine, 14(1), 83-94.

[36] Naranjo, D. M., Fisher, F. L., Areán, P. A., Hessler, D., \& Mullan, J. (2011). Patients with Type 2 Diabetes at Risk for Major Depressive Disorder Over Time. Annals of Family Medicine, 9, 115-120.

[37] National Diabetes Association of Ghana (2012, June 14). Ghana has 4m Diabetics. Daily Guide.

[38] Park, J., Hong, J. P., Park, S., \& Cho, M. (2012). The relationship between religion and mental disorders in a Korean population. Psychiatry Investigation, 9(1), 29-35

[39] Paddison, C. A. M. (2010). Exploring physical and psychological wellbeing among adults with Type 2 diabetes in New Zealand: identifying a need to improve the experiences of Pacific peoples. The New Zealand Medical Journal, 123(1310), 30-42.

[40] Paddison, C. A. M., Alpass, F. M., \& Stephens, C. V. (2010). Using the Common Sense Model of illness self-regulation to understand diabetes-related distress: The importance of being able to 'make sense' of diabetes. New Zealand Journal of Psychology, 39(1), 45-50.
[41] Petriček, G., Vrcić-Keglević, M., Vuletić, G., Cerovečki, V., Ožvačić, Z., \& Murgić, L. (2009). Illness Perception and Cardiovascular Risk Factors in Patients with Type 2 Diabetes: Cross-sectional Questionnaire Study. Croatia Medical Journal, 50(6), 583-593.

[42] Peyrot, M., Rubin, R.R., Lauritzen, T., Snoek, F. J., Matthews, D. R., \& Skovlund, S. E. (2005). Psychosocial problems and barriers to improved diabetes management: Results of the cross-national Diabetes Attitudes, Wishes and Needs (DAWN) Study.

[43] Rees, G., Fry, A., Cull, A., \& Sutton, S. (2004). Illness perceptions and distress in women at increased risk of breast cancer. Psychology and Health, 19 (6), 749-765.

[44] Roupa, Z., Koulouri, A., Sotiropoulou, P., Makrinika, E., Marneras, X., Lahana, I., \& Gourni, M. (2009). Anxiety and depression in patients with Type 2 Diabetes Mellitus, depending on sex and body mass index. Health Science Journal, 3(1), 32-40.

[45] Scheier, M. F., \& Carver, C. S. (1985). Optimism, coping, and health: Assessment and implications of generalized outcome expectancies. Health Psychology, 4(3), 219-47.

[46] Shaw, J., Sicree, R., \& Zimmet, P. (2010). Global estimates of the prevalence of diabetes for 2010 and 2030. Diabetes Research and Clinical Practice, 87(1), 4-14.

[47] Tsang, J., \& McCullough, M. E. (2003). Measuring religious constructs: A hierarchical approach to construct organization and scale selection. In S.J. Lopez, \& C.R. Snyder (Eds.). Positive psychological assessment: A handbook of models and measures (pp.345-360). Washington, DC: American Psychological Association.

[48] Tuncay, T., Musabak, I., Gok, D. E., \& Kutlu, M. (2008). The relationship between anxiety, coping strategies and characteristics of patients with diabetes. Health and Quality of Life Outcomes, 6, 79-87. 